\title{
Potencial produtivo de videiras cultivadas sob cobertura de plástico
}

\author{
Geraldo Chavarria( ${ }^{(1)}$, Henrique Pessoa dos Santos (2), Francisco Mandelli(2), Gilmar Arduino Bettio Marodin ${ }^{(1)}$, \\ Homero Bergamaschi(1) e Loana Silveira Cardoso ${ }^{(1)}$
}

\begin{abstract}
(1)Universidade Federal do Rio Grande do Sul, Departamento de Horticultura e Silvicultura, Caixa Postal 776, CEP 91540-000 Porto Alegre, RS. E-mail: geraldochavarria@hotmail.com, marodin@ufrgs.br, homerob@ufrgs.br, loanacar@yahoo.com.br (2)Embrapa Uva e Vinho, Caixa Postal 130, CEP 95700-000 Bento Gonçalves, RS. E-mail: henrique@cnpuv.embrapa.br, mandelli@cnpuv.embrapa.br
\end{abstract}

Resumo - O objetivo deste trabalho foi avaliar a influência do uso de cobertura de plástico sobre os componentes do rendimento da videira (Vitis vinifera L.) cultivar Moscato Giallo. O experimento foi realizado nas safras 2005/2006 e 2006/2007, em Flores da Cunha, RS, em duas áreas de vinhedo, uma com cobertura de plástico impermeável e outra sem cobertura (controle). O microclima foi avaliado quanto à temperatura e umidade relativa do ar, radiação fotossinteticamente ativa e velocidade do vento próximo ao dossel vegetativo e aos cachos. A avaliação dos componentes de rendimento ocorreu em delineamento experimental inteiramente ao acaso, e foram identificadas dez plantas marcadas aleatoriamente em cada área. Avaliaram-se a produção por planta e por hectare, o número de cachos por planta e por metro quadrado, o número de sarmentos por metro quadrado, a massa e comprimento de cacho, a massa de engaço, o número de bagas por cacho, o diâmetro transversal de bagas e a relação entre massa de película e massa de polpa. A cobertura de plástico possibilita aumento na produtividade, não afeta a relação entre massa de casca e massa de polpa das bagas e favorece a estabilidade de produção, independentemente das condições meteorológicas no ciclo.

Termos para indexação: Vitis vinifera, microclima, produtividade, sistema de produção.

\section{Yield potential of grapevine cultivated under plastic cover}

\begin{abstract}
The objective of this work was to evaluate the effect of plastic cover on the yield components of grapevine (Vitis vinifera L.) cultivar Moscato Giallo. The experiment was carried out in 2005/2006 and 2006/2007 crop seasons, in Flores da Cunha, RS, Brazil, in two vineyard areas, one covered with an impermeable plastic film and other without covering (control). The microclimate was evaluated in terms of air temperature, air relative humidity, photosynthetically active radiation and wind speed above canopy and close to clusters. The yield components were evaluated in a completely randomized design, in ten plants randomly selected in each area. Measures were made for production per plant, yield per hectare, clusters per vine, clusters per square meter, twigs per square meter, weight and length of clusters, weight of rachis, number of berries per cluster, diameter of berries, and skim/pulp ratio. Plastic cover promotes yield increases, does not affect pulp/skin ratio, and promotes production stability, independently of climate conditions in each season.
\end{abstract}

Index terms: Vitis vinifera, microclimate, yield, production system.

\section{Introdução}

A produtividade da videira (Vitis vinifera L.) pode ser afetada por diversos aspectos, tais como o clima, práticas culturais, nutrição mineral, disponibilidade hídrica, pragas e doenças. A influência que o ambiente exerce sobre a videira é resultado da interação desses fatores, cujo efeito total está relacionado com o potencial genético de resposta às condições do ambiente, o que determina sua produtividade e a qualidade dos frutos produzidos (Feldberg et al., 2007).

Entre os fatores ambientais que influenciam o crescimento e o desenvolvimento das plantas estão os de ação direta, como radiação solar, temperatura e água no solo, e os de ação indireta, como latitude, longitude e textura do solo (Alvim, 1962). O microclima constitui-se um dos aspectos de maior relevância no caráter produtivo da cultura, por afetar os fatores de ação direta (Pedro Júnior et al., 2007a).

$\mathrm{O}$ microclima corresponde às condições climáticas em superfícies pequenas. De acordo com Carbonneau (1984), existem dois tipos de microclima: o natural que corresponde a superfícies da ordem de 10 a $100 \mathrm{~m}^{2}$ - e o microclima da planta - que é caracterizado por variáveis climáticas entorno de cada planta. 
A forma como a videira é conduzida, considerando o manejo do vinhedo, define o rendimento e a qualidade dos frutos (Manfroi et al., 2004) e tem influência direta sobre a atividade metabólica da planta (Haselgrove et al., 2000). No manejo destacam-se os cuidados com a quantidade e a disposição das folhas no dossel vegetativo, pois condicionam a interceptação luminosa, da qual a produtividade de matéria seca é dependente (Weyand \& Schultz, 2006).

A cobertura de plástico é uma tecnologia recente na vitivinicultura brasileira e tem sido empregada para minimizar os efeitos do clima durante as safras (Mota et al., 2008), sobretudo pela redução da água livre sobre folhas e cachos, o que diminui a incidência de doenças fúngicas e a necessidade de pulverizações (Chavarria et al., 2007), e a redução dos danos causados por granizo e ventos fortes (Chavarria, 2008). A cobertura de plástico promove alterações nas variáveis do microclima, principalmente na temperatura, na radiação, nos ventos e na presença de água livre sobre as folhas (Cardoso et al., 2008). Essas alterações podem modificar as respostas fisiológicas da videira, sendo, em alguns casos, fator atenuante de estresses hídricos e promotor de melhores condições para o crescimento da planta (Chavarria et al., 2008).

Entre as principais interferências da cobertura de plástico no microclima da videira destaca-se a restrição da radiação solar, cujas intensidade (Rana et al., 2004) e qualidade são reduzidas, com redução na faixa do ultravioleta e do azul e na relação do vermelho/ vermelho distante (Chavarria, 2008). Essas alterações podem afetar diretamente o potencial de produção e o crescimento das plantas, pois com a redução da radiação pode-se ter uma restrição no processo fotossintético das plantas e na fertilidade das gemas (López-Miranda, 2002).

O objetivo deste trabalho foi avaliar a influência da cobertura de plástico sobre os componentes do rendimento da cultivar Moscato Giallo.

\section{Material e Métodos}

O experimento foi conduzido em 2005/2006 e 2006/2007, em vinhedo comercial localizado em Flores da Cunha, RS, $\left(29^{\circ} 6^{\prime} \mathrm{S}\right.$, e $51^{\circ} 20^{\prime} \mathrm{W}$ e altitude de $541 \mathrm{~m}$ ), com a cultivar Moscato Giallo (clone VCR1), enxertada em porta-enxerto Kobber 5BB, plantada com espaçamento de $3 \times 0,9 \mathrm{~m}$.
As plantas foram conduzidas em "Y", com fileiras de 35 m, na direção Nordeste-Sudoeste, com poda mista, com quatro varas de seis a oito gemas e seis esporões de duas gemas em cada planta. $\mathrm{O}$ vinhedo foi dividido em duas partes. A primeira foi constituída por 12 fileiras cobertas na linha de cultivo por lonas de polipropileno transparentes, trançadas e impermeabilizadas com polietileno de baixa densidade, com $160 \mu \mathrm{m}$ de espessura e $2,65 \mathrm{~m}$ de largura. $\mathrm{Na}$ segunda parte, foram mantidas cinco fileiras descobertas, cujas linhas centrais foram consideradas como plantas-controle.

Em 2/3/2006 e 12/2/2007, em delineamento completamente casualizado, foram identificadas, aleatoriamente, dez plantas em cada área. Nessas plantas avaliaram-se a produção por planta, a produção por hectare, o número de cachos por planta e por metro quadrado, o número de sarmentos por metro quadrado, a massa e o comprimento de cacho, a massa de engaço, o número de bagas por cacho e o diâmetro transversal de bagas. Nas duas safras foram coletadas de 50 bagas, com três repetições por área, e avaliada a relação entre a massa de casca e a massa de polpa. Paras as avaliações de componentes do rendimento, foi utilizada balança digital (modelo Mettler PC 4400, marca Deltarange) e paquímetro digital (marca Digimess).

Desde a poda até 30 dias após a colheita, o microclima foi avaliado por meio de medições de temperatura e umidade relativa do ar (psicrômetros de pares termoelétricos), velocidade do vento (anemômetro de conchas) e radiação fotossinteticamente ativa (RFA: 400-700 nm, com o emprego de barras de 1,20 m, equipadas com cinco células fotovoltaicas ligadas em série), na altura do dossel vegetativo e dos cachos. Os sensores foram conectados a sistemas automáticos de aquisição de dados (CR10 e CR21X, Campbell). Ambos os sistemas foram programados para efetuar leituras a cada minuto e calcular a média em intervalos de 30 minutos.

$\mathrm{Na}$ análise estatística utilizou-se o programa $\mathrm{R}$ (R-Project, 2007). Os dados de microclima foram pareados, e as diferenças entre tratamentos foram analisadas por regressão linear em função da área descoberta. A significância do coeficiente angular das equações resultantes foi avaliada pelo teste $t$. $\mathrm{Na}$ análise dos dados de radiação e velocidade do vento, o intercepto foi fixado em zero, pois quando a medida externa é nula, a interna deve também ser nula. As variáveis dos componentes de rendimento 
foram submetidas à análise de variância (ANOVA) e as médias comparadas pelo teste de Tukey, a 5\% de probabilidade.

\section{Resultados e Discussão}

A cobertura de plástico proporcionou mudanças no microclima do vinhedo em comparação ao cultivo a céu aberto. A cobertura aumentou a temperatura diurna próxima ao dossel vegetativo, não influenciou a umidade relativa do ar e diminuiu a RFA e a velocidade do vento (Tabela 1). Esses efeitos da cobertura de plástico sobre o microclima da videira também foram reportados por Cardoso et al. (2008).

Com relação à temperatura, as máximas diárias foram as que mais sofreram influência da cobertura. À medida que a temperatura máxima fora da cobertura diminuiu, a redução correspondente sob a cobertura foi menor. Isso pode ser verificado pelo coeficiente de regressão altamente significativo $\left(r^{2}=0,74 ; p<0,001\right)$ que mostra que, para cada grau centígrado de redução na temperatura externa, a temperatura abaixo da cobertura caiu $0,9^{\circ} \mathrm{C}$. Segovia et al. (1997) atribuíram essa menor variação térmica à retenção de ar e, consequentemente, ao maior acúmulo térmico diário no ambiente protegido. Isto ficou evidenciado, pois a velocidade do vento foi atenuada em $90,4 \%\left(r^{2}=0,49 ; p<0,0001\right)$ junto ao dossel vegetativo das plantas sob cobertura (Tabela 1). Para a videira, temperaturas mais elevadas podem promover melhor frutificação e desenvolvimento das bagas. Cultivares de espécies de videira americanas têm maior fertilidade em temperaturas mais baixas (21 a $\left.22^{\circ} \mathrm{C}\right)$ do que cultivares da espécie Vitis vinifera $(27$ a $28^{\circ} \mathrm{C}$ ) (Mullins et al., 1992). Segundo Coombe (1995), existe um período considerado mais crítico (estádios 13 a 18) em que a planta necessita de temperaturas mais elevadas, associadas à maior disponibilidade de radiação solar.

A RFA sob a cobertura sofreu redução de $37 \%$ $\left(\mathrm{r}^{2}=0,91, \mathrm{p}<0,0001\right)$. O efeito variou de um ciclo para outro, tendo uma redução de $32,59 \%$ no primeiro ciclo e de $43,39 \%$ no segundo ciclo, o que está relacionado à perda de transparência do plástico ao longo do tempo (Tabela 1). Rana et al. (2004) também observaram diminuição da RFA em $17 \%$ sob clarite e em $32 \%$ em áreas com cobertura de plástico impermeável e translúcido.

Na safra 2006, as plantas cobertas e descobertas não apresentaram diferenças significativas na maioria dos componentes do rendimento avaliados. Entretanto, a área com cobertura apresentou produtividade superior, embora sem diferenças significativas em relação à área descoberta (Tabela 2). Essa diferença pode ser atribuída às variáveis de comprimento de cachos e número e massa de bagas, as quais foram maiores na área coberta (Tabela 3).

A semelhança de produtividade na primeira safra, entre os dois sistemas de produção, deve-se ao fato de que, anteriormente ao experimento, toda a área estava com cobertura de plástico, que foi retirada em parte da área para servir como testemunha. Como os processos de indução e diferenciação dos primórdios florais da videira são definidos no ano anterior (Boss et al., 2003), não foi observada influência da cobertura na produtividade das plantas no primeiro ano de avaliação. Entretanto, na safra 2007, a produção por

Tabela 1. Médias das observações microclimáticas em vinhedo da cultivar Moscato Giallo, conduzida em "Y" com (C) e sem (D) cobertura de plástico, nos ciclos 2005/2006 e 2006/2007, em Flores da Cunha, RS.

\begin{tabular}{|c|c|c|c|c|c|}
\hline \multirow[t]{2}{*}{ Variáveis microclimáticas } & \multicolumn{2}{|c|}{$2005 / 2006$} & \multicolumn{2}{|c|}{$2006 / 2007$} & \multirow[t]{2}{*}{ Equações de regressão ${ }^{(2)}$} \\
\hline & $\mathrm{C}$ & $\mathrm{D}$ & $\mathrm{C}$ & $\mathrm{D}$ & \\
\hline Temperatura do ar máxima $-\operatorname{dossel}\left({ }^{\circ} \mathrm{C}\right)$ & 31,94 & 28,19 & 30,37 & 31,18 & $\mathrm{C}=4,51+0,897 \mathrm{D}\left(\mathrm{r}^{2}=0,74\right)$ \\
\hline Temperatura do ar média $-\operatorname{dossel}\left({ }^{\circ} \mathrm{C}\right)$ & 21,28 & 20,28 & 21,91 & 21,84 & $\mathrm{C}=1,37+0,960 \mathrm{D}\left(\mathrm{r}^{2}=0,95^{*}\right)$ \\
\hline Temperatura do ar mínima $-\operatorname{dossel}\left({ }^{\circ} \mathrm{C}\right)$ & 14,62 & 14,45 & 16,33 & 16,07 & $\mathrm{C}=0,64+0,972 \mathrm{D}\left(\mathrm{r}^{2}=0,94 * *\right)$ \\
\hline Umidade relativa do ar - dossel (\%) & 83,12 & 82,51 & -(1) & - & $\mathrm{C}=13,2+0,848 \mathrm{D}\left(\mathrm{r}^{2}=0,89\right)$ \\
\hline Umidade relativa do ar - cachos $(\%)$ & 83,84 & 84,87 & - & - & $\mathrm{C}=23,1+0,719 \mathrm{D}\left(\mathrm{r}^{2}=0,74\right)$ \\
\hline $\begin{array}{l}\text { Radiação fotossinteticamente ativa - dossel } \\
\left(\mu \mathrm{mol} \text { fótons } \mathrm{m}^{-2} \text { por dia) }\right.\end{array}$ & 284,44 & 421,90 & 228,39 & 403,39 & $\mathrm{C}=0,629 \mathrm{D}\left(\mathrm{r}^{2}=0,91\right)$ \\
\hline $\begin{array}{l}\text { Radiação fotossintenticamente ativa }- \text { cachos } \\
\text { (umol fótons } \mathrm{m}^{-2} \text { por dia) }\end{array}$ & 66,08 & 151,73 & 66,61 & 87,23 & $\mathrm{C}=0,570 \mathrm{D}\left(\mathrm{r}^{2}=0,80\right)$ \\
\hline Velocidade do vento diária $\left(\mathrm{m} \mathrm{s}^{-1}\right)$ & 0,093 & 0,904 & 0,079 & 0,817 & $\mathrm{C}=0,107 \mathrm{D}\left(\mathrm{r}^{2}=0,49\right)$ \\
\hline
\end{tabular}

${ }^{(1)}$ Ausência de dados por problemas nos sensores (termopares de bulbo úmido). ${ }^{(2)}$ Equações de regressão referentes à análise das variáveis microclimáticas dos dois ciclos em conjunto, por não haver diferença significativa na comparação entre os anos safras. * e **Coeficiente angular significativo a 5 e $1 \%$ de probabilidade, respectivamente, pelo teste $\mathrm{t}$. 
planta e, consequentemente, a produtividade foram $39 \%$ maiores na área com cobertura. A diferença se deve ao fato de as plantas do cultivo descoberto terem diminuído a produção, tanto nas varas como nos esporões, de forma que o rendimento por hectare do cultivo descoberto diminuiu $27 \%$ em relação ao ciclo anterior (Tabela 2). Contudo, as plantas cobertas não apresentaram aumento de produtividade por planta em comparação à observada no ciclo anterior.

O diferencial de produtividade na safra 2007, entre os tratamentos, não foi ocasionado pelo aumento da massa média dos cachos, que foi de 222,43 $\mathrm{g}$ na área coberta e 213,54 g na área descoberta (Tabela 3). A maior produtividade em plantas cobertas está relacionada ao maior número de cachos. De forma geral, também foi observado um aumento de $17 \%$ no número de cachos por metro quadrado nas áreas com cobertura na safra 2007, comparado ao ciclo anterior (Tabela 2). Este resultado não foi obtido em razão do aumento da carga de gemas, mas pela maior brotação das gemas. Salienta-se que o tipo de poda e a quantidade de gemas por planta (em torna de 40 gemas) foram mantidos iguais nos dois ciclos. Portanto, esta maior brotação das gemas pode estar relacionada a um maior aporte de reservas, haja vista o maior período de atividade biológica das folhas que o cultivo protegido propicia. Chavarria (2008) observou que as folhas de videiras cobertas permaneceram realizando fotossíntese por um período superior a 21 dias, comparado às videiras em cultivo convencional, devido à melhor condição fitossanitária do sistema de cultivo coberto.

Alguns trabalhos realizados com a cultivar Cabernet Sauvignon apontam que a cobertura de plástico não afeta a produtividade (Ferreira et al., 2004; Mota et al., 2008). Todavia, Detoni et al. (2007), ao utilizar a cobertura sobre as plantas e Pedro Júnior et al. (2007b), ao utilizar a cobertura individual dos cachos, observaram aumentos nas produtividades das cultivares Cabernet Sauvignon. Palma et al. (1999) destacaram que a cultivar Matilde apresentou maior rendimento quando cultivada sob cobertura de plástico na Itália. Contudo, deve-se enfatizar que o desempenho agronômico sob cobertura de plástico é dependente da cultivar utilizada (Novello \& Palma, 2008). A cultivar Moscato Giallo, utilizada no presente trabalho, é bastante produtiva em cultivo convencional e pode apresentar ainda um melhor desempenho agronômico sob cobertura de plástico.

Da mesma forma que o excesso de radiação solar pode causar danos aos frutos, a sua restrição pode afetar o processo fotossintético, o crescimento e o acúmulo de reservas e acarretar baixa frutificação no ciclo posterior (Mullins et al., 1992). Sabe-se que as plantas sob restrição luminosa buscam meios para compensar essa falta, e utilizam mecanismos como o aumento de superfície foliar total e de volume da camada paliçádica das folhas, para aumentar o número de pigmentos receptores de luz (Wheller \& Fagerberg, 2000). As folhas sombreadas podem apresentar maior absorção de fótons, a fim de maximizar a assimilação de carbono e a eficiência do uso de nutrientes sob condições de suprimento energético limitado pelas adaptações anatômicas, morfológicas e bioquímicas (Henry \& Aarssen, 1997). No aspecto bioquímico, essas diferenças de taxas fotossintéticas podem ser atribuídas à maior frequência por unidade de área de ribulose

Tabela 2. Componentes do rendimento em videiras (Vitis vinifera) da cultivar Moscato Giallo, conduzida em "Y" com (C) e sem (D) cobertura de plástico, nos anos safras 2005/2006 e 2006/2007, em Flores da Cunha, $\mathrm{RS}^{(1)}$.

\begin{tabular}{|c|c|c|c|c|c|c|c|c|c|c|}
\hline \multirow[t]{2}{*}{ Parte da planta } & \multicolumn{2}{|c|}{ Produção por planta $(\mathrm{kg})$} & \multicolumn{2}{|c|}{ Produtividade $\left(\mathrm{Mg} \mathrm{ha}^{-1}\right)$} & \multicolumn{2}{|c|}{ Cachos por planta } & \multicolumn{2}{|c|}{ Cachos por $\mathrm{m}^{2}$} & \multicolumn{2}{|c|}{ Ramos por $\mathrm{m}^{2}$} \\
\hline & $\mathrm{C}$ & $\mathrm{D}$ & $\mathrm{C}$ & $\mathrm{D}$ & $\mathrm{C}$ & $\mathrm{D}$ & $\mathrm{C}$ & $\mathrm{D}$ & $\mathrm{C}$ & $\mathrm{D}$ \\
\hline & \multicolumn{10}{|c|}{ Ano safra $2005 / 2006$} \\
\hline Esporão & $4,12 \mathrm{a}$ & $2,51 \mathrm{a}$ & $15,26 \mathrm{a}$ & $9,3 \mathrm{a}$ & $18,1 \mathrm{a}$ & $11,78 \mathrm{a}$ & $6,7 \mathrm{a}$ & $4,36 a$ & - & - \\
\hline Vara & $7,00 \mathrm{a}$ & $6,79 a$ & $25,93 \mathrm{a}$ & $25,15 \mathrm{a}$ & $23,5 \mathrm{a}$ & $28,11 \mathrm{a}$ & $8,7 \mathrm{a}$ & $10,41 \mathrm{a}$ & - & - \\
\hline Total & $11,12 \mathrm{a}$ & $9,3 a$ & $41,19 a$ & $34,44 a$ & $41,6 \mathrm{a}$ & $39,89 \mathrm{a}$ & $15,5 \mathrm{a}$ & $14,77 \mathrm{a}$ & $10,51 \mathrm{a}$ & $8,88 \mathrm{a}$ \\
\hline \multirow[t]{2}{*}{$\mathrm{CV}(\%)$} & \multicolumn{2}{|c|}{41,18} & \multicolumn{2}{|c|}{41,18} & \multicolumn{2}{|c|}{32,91} & \multicolumn{2}{|c|}{32,91} & \multicolumn{2}{|c|}{18,25} \\
\hline & \multicolumn{10}{|c|}{ safra $2006 / 2007$} \\
\hline Esporão & $4,95 \mathrm{a}$ & $3,10 \mathrm{~b}$ & $18,33 \mathrm{a}$ & $11,48 b$ & $25,78 \mathrm{a}$ & $15,22 b$ & $9,55 \mathrm{a}$ & $5,64 b$ & - & - \\
\hline Vara & $6,23 \mathrm{a}$ & $3,71 \mathrm{~b}$ & $23,07 \mathrm{a}$ & $13,74 \mathrm{~b}$ & $24,78 \mathrm{a}$ & $17,11 \mathrm{a}$ & $9,18 \mathrm{a}$ & $6,34 \mathrm{a}$ & - & - \\
\hline Total & $11,19 \mathrm{a}$ & $6,81 \mathrm{~b}$ & $41,44 a$ & $25,22 \mathrm{~b}$ & $50,56 a$ & $32,33 b$ & $18,73 \mathrm{a}$ & $11,98 b$ & $9,66 \mathrm{a}$ & $10,81 \mathrm{a}$ \\
\hline CV (\%) & \multicolumn{2}{|c|}{34,93} & \multicolumn{2}{|c|}{34,93} & \multicolumn{2}{|c|}{38,43} & \multicolumn{2}{|c|}{38,43} & \multicolumn{2}{|c|}{19,75} \\
\hline
\end{tabular}

${ }^{(1)}$ Médias seguidas de letras iguais, na linha, para cada componente de rendimento, não diferem entre si pelo teste de Tukey, a $5 \%$ de probabilidade. 
bifosfato carboxilase/oxigenase, de citocromos, de fotossistemas I e II, de centros de reação e de enzimas ligadas à respiração (Noguchi et al., 1996).

O processo fotossintético é beneficiado pelo microclima propiciado pela cobertura (Chavarria, 2008), e isto reflete-se no aumento da produtividade (Tabela 2). Sabe-se que a cobertura de plástico pode atenuar estresses oriundos de excesso de radiação solar ou de falta de água, pois reduz a radiação solar e a velocidade do vento e causa decréscimo na demanda evaporativa (Chavarria, 2008). Neste caso, favorece a fotossíntese e influencia positivamente a produção (Intrigliolo \& Castel, 2008). Chavarria et al. (2008), ao estudar ciclos diários de trocas gasosas em condições de alta radiação solar, demonstraram que videiras cobertas podem realizar fotossíntese em períodos em que as plantas descobertas estão inibidas pelo estresse hídrico.

A diminuição da RFA na área coberta teve reflexo sobre o comprimento dos cachos nas duas safras e aumentou o crescimento estiolado do ráquis (Tabela 3 ). Em razão disso, o engaço também apresentou massa superior no cultivo coberto. Destaca-se também, que os cachos da área coberta apresentaram maior número de bagas nas duas safras. Entretanto, diferenças significativas foram observadas somente na segunda safra, quando o número médio de bagas por cacho foi de 118 e 88 , respectivamente, nas áreas coberta e descoberta. Este resultado pode estar relacionado à melhor frutificação, devido às condições do microclima proporcionado pela cobertura, já que no subperíodo fenológico que vai do florescimento ao estádio de chumbinho da baga, os excessos de chuva e vento afetam a polinização e o pegamento dos frutos (Mullins et al., 1992). Contudo, nos dois ciclos estudados não houve excesso de chuvas e de vento nesses estádios fenológicos. Porém, como pode ser observado na caracterização microclimática (Tabela 1), a cobertura de plástico reduziu consideravelmente a velocidade do vento, o que, mesmo em condições meteorológicas normais da Serra Gaúcha, pode reduzir a queda de flores e favorecer o maior número de bagas por cacho.

A disponibilidade hídrica para as plantas na área coberta tende a ser maior. Embora haja uma restrição hídrica na linha de cultivo, as videiras alocam seu sistema radicular para a entrelinha, onde ocorre maior concentração de água, comparativamente ao cultivo descoberto (Chavarria, 2008). Associado a isto, a redução da radiação solar e do vento possibilita menor demanda evaporativa e estimula maior abertura estomática (Chavarria et al., 2008). Esse fato pode influenciar positivamente o crescimento das bagas, devido à maior quantidade de água favorecer à pressão de turgor, que é responsável pelo crescimento celular (Ojeda et al., 2004). Todavia, somente foram observadas diferenças significativas na massa das bagas da primeira safra e no diâmetro transversal da segunda safra e nesta safra as bagas apresentaramse menores (Tabela 3). Isso demonstra que o efeito atenuador da cobertura sobre a condição hídrica pode ocorrer em anos com estresses hídricos severos, diferentemente das condições ocorridas nos anos safras avaliados.

$\mathrm{O}$ número de sementes e a relação de massas casca/polpa não foram afetados pela cobertura de plástico nos dois ciclos estudados (Tabela 4). Na safra 2005/2006, a massa total e a massa da casca não foram influenciadas pela cobertura. Entretanto, na safra 2006/2007, a massa da casca das bagas da

Tabela 3. Características de cachos de videiras (Vitis vinifera) da cultivar Moscato Giallo, conduzida em "Y" com (C) e sem (D) cobertura de plástico, nos anos safras 2005/2006 e 2006/2007, em Flores da Cunha, RS ${ }^{(1)}$.

\begin{tabular}{|c|c|c|c|c|c|c|c|c|c|c|c|c|}
\hline \multirow[t]{2}{*}{$\begin{array}{l}\text { Parte da } \\
\text { planta }\end{array}$} & \multicolumn{2}{|c|}{$\begin{array}{c}\text { Massa fresca de } \\
\text { cacho }(\mathrm{g})\end{array}$} & \multicolumn{2}{|c|}{$\begin{array}{l}\text { Comprimento de } \\
\text { cachos }(\mathrm{cm})\end{array}$} & \multicolumn{2}{|c|}{$\begin{array}{c}\text { Massa fresca de } \\
\text { engaço }(\mathrm{g})\end{array}$} & \multicolumn{2}{|c|}{$\begin{array}{l}\text { Número bagas por } \\
\text { cacho }\end{array}$} & \multicolumn{2}{|c|}{$\begin{array}{c}\text { Diâmetro bagas } \\
(\mathrm{mm})\end{array}$} & \multicolumn{2}{|c|}{$\begin{array}{c}\text { Massa fresca de } \\
\text { bagas }(\mathrm{g})\end{array}$} \\
\hline & $\mathrm{C}$ & $\mathrm{D}$ & $\mathrm{C}$ & $\mathrm{D}$ & $\mathrm{C}$ & $\mathrm{D}$ & $\mathrm{C}$ & $\mathrm{D}$ & $\mathrm{C}$ & $\mathrm{D}$ & $\mathrm{C}$ & $\mathrm{D}$ \\
\hline & \multicolumn{12}{|c|}{ Ano safra $2005 / 2006$} \\
\hline Esporão & $206,55 a$ & $211,61 \mathrm{a}$ & $15,33 \mathrm{a}$ & $12,81 \mathrm{~b}$ & $11,16 \mathrm{a}$ & $13,03 a$ & $95,5 \mathrm{a}$ & $98,2 b$ & - & - & $2,90 \mathrm{a}$ & $2,43 b$ \\
\hline Vara & $297,19 a$ & $241,41 \mathrm{a}$ & $18,66 \mathrm{a}$ & $13,67 b$ & $15,3 \mathrm{a}$ & $12,57 \mathrm{a}$ & $118,2 \mathrm{a}$ & $94,7 \mathrm{~b}$ & - & - & $2,73 \mathrm{a}$ & $2,01 \mathrm{~b}$ \\
\hline Média & $251,86 a$ & $226,51 a$ & $16,99 a$ & $13,24 b$ & $13,24 a$ & $12,81 \mathrm{a}$ & $105,85 a$ & $96,45 b$ & $16,95 a$ & $16,50 \mathrm{a}$ & $2,81 \mathrm{a}$ & $2,22 b$ \\
\hline \multirow[t]{2}{*}{ CV $(\%)$} & \multicolumn{2}{|c|}{22,38} & \multicolumn{2}{|c|}{14,62} & \multicolumn{2}{|c|}{32,42} & \multicolumn{2}{|c|}{32,16} & & & \multicolumn{2}{|c|}{22,51} \\
\hline & \multicolumn{12}{|c|}{ Ano safra $2006 / 2007$} \\
\hline Esporão & $194,65 \mathrm{a}$ & $203,10 a$ & $17,55 \mathrm{a}$ & $14,67 b$ & $10,49 \mathrm{a}$ & $8,59 \mathrm{~b}$ & $90,25 \mathrm{a}$ & $79,25 b$ & - & - & $2,61 \mathrm{a}$ & $2,57 \mathrm{a}$ \\
\hline Vara & $250,21 \mathrm{a}$ & $223,97 \mathrm{a}$ & $18,52 \mathrm{a}$ & $16,04 \mathrm{~b}$ & $15,45 \mathrm{a}$ & $9,84 \mathrm{~b}$ & $145,4 \mathrm{a}$ & $96,58 \mathrm{~b}$ & - & - & $2,30 \mathrm{a}$ & $2,41 \mathrm{a}$ \\
\hline Média & $222,43 a$ & $213,54 a$ & $18,03 \mathrm{a}$ & $15,35 b$ & $12,97 \mathrm{a}$ & $9,20 \mathrm{~b}$ & $117,83 a$ & $87,69 b$ & $16,04 \mathrm{~b}$ & $17,03 \mathrm{a}$ & $2,48 \mathrm{a}$ & $2,49 a$ \\
\hline CV $(\%)$ & \multicolumn{2}{|c|}{18,63} & \multicolumn{2}{|c|}{14,39} & \multicolumn{2}{|c|}{37,68} & \multicolumn{2}{|c|}{35,70} & & & \multicolumn{2}{|c|}{25,50} \\
\hline
\end{tabular}

(1)Médias seguidas de letras iguais, na linha, para cada variável, não diferem entre si pelo teste de Tukey, a 5\% de probabilidade. 
Tabela 4. Características das bagas de videiras (Vitis vinifera) da cultivar Moscato Giallo, conduzida em "Y" com (C) e sem (D) cobertura de plástico, nos anos safras 2005/2006 e 2006/2007, em Flores da Cunha, RS $(n=50)^{(1)}$.

\begin{tabular}{lrrrrrrr}
\hline Componentes & \multicolumn{3}{c}{ Safra 2005/2006 } & & \multicolumn{3}{c}{ Safra 2006/2007 } \\
\cline { 2 - 3 } \cline { 6 - 8 } da baga & \multicolumn{1}{c}{$\mathrm{C}$} & \multicolumn{1}{c}{$\mathrm{D}$} & $\mathrm{CV}(\%)$ & & $\mathrm{C}$ & $\mathrm{D}$ & $\mathrm{CV}(\%)$ \\
\hline Massa total $(\mathrm{g})$ & $138,11 \mathrm{a}$ & $136,42 \mathrm{a}$ & 9,15 & & $159,44 \mathrm{a}$ & $149,52 \mathrm{~b}$ & 8,77 \\
Casca $(\mathrm{g})$ & $38,28 \mathrm{a}$ & $40,75 \mathrm{a}$ & 9,36 & & $43,56 \mathrm{a}$ & $32,61 \mathrm{~b}$ & 9,74 \\
Número de & $3,89 \mathrm{a}$ & $4,02 \mathrm{a}$ & 10,18 & & $3,44 \mathrm{a}$ & $3,24 \mathrm{a}$ & 9,96 \\
sementes & & & & & & \\
Relação & $2,65 \mathrm{a}$ & $2,40 \mathrm{a}$ & 17,45 & & $2,58 \mathrm{a}$ & $3,48 \mathrm{a}$ & 19,20
\end{tabular}

casca/polpa

${ }^{(1)}$ Médias seguidas de letras iguais, na linha, para cada safra, não diferem entre si pelo teste de Tukey, a $5 \%$ de probabilidade.

área coberta foi significativamente maior. Em uvas destinadas à vinificação é desejada maior quantidade de casca, pois é nela que está a maior quantidade de compostos que incrementam a qualidade enológica do vinho, como antocianinas e taninos - em uvas para vinho tinto (Ojeda et al., 2004) - e precursores aromáticos, como terpenos - em uvas para vinhos brancos (Karagiannis et al., 2000). Isto é importante para a cultivar Moscato Giallo, que é conhecida pela sua característica aromática pronunciada (Schneider et al., 2001).

\section{Conclusões}

1. A cobertura de plástico impermeável sobre a linhas de cultivo da videira cultivar Moscato Giallo aumenta a produtividade e favorece a estabilidade da produção entre os ciclos.

2. A cobertura de plástico não afeta a relação das massas de casca e polpa das bagas da videira cultivar Moscato Giallo.

\section{Referências}

ALVIM, P.T. Los factores de la productividad agrícola. In: CURSO INTERNATIONAL DE BASES FISIOLÓGICAS DE LA PRODUCCIÓN AGRÍCOLA. Lima: Instituto Interamericano de Ciencias Agrícolas, 1962. p.1-20.

BOSS, P.K.; BUCKERIDGE, E.J.; POOLE, A.; THOMAS, M.R. New insights into grapevine flowering. Functional Plant Biology, v.30, p.593-606, 2003.

CARBONNEAU, A. Place du microclimat de la partie aérienne parmi les facteurs déterminant les productions viticoles. Bulletin de l'OIV, v.57, p.473-477, 1984.

CARDOSO, L.S.; BERGAMASCHI, H.; COMIRAM, F.; CHAVARRIA, G.; MARODIN, G.A.B.; DALMAGO, G.A.; SANTOS,H.P. dos; MANDELLI,F. Alteraçõesmicrometeorológicas em vinhedos pelo uso de coberturas de plástico. Pesquisa Agropecuária Brasileira, v.43, p.441-447, 2008.
CHAVARRIA, G. Ecofisiologia e fitotecnia do cultivo protegido de videiras cv. Moscato Giallo (Vitis vinifera L.). 2008. 136p. Tese (Doutorado) - Universidade Federal do Rio Grande do Sul, Porto Alegre.

CHAVARRIA, G.; SANTOS, H.P. dos; FELIPPETO, J.; MARODIN, G.A.B.; BERGAMASCHI, H.; CARDOSO, L.S.; FIALHO, F.B. Relações hídricas e trocas gasosas em vinhedo sob cobertura plástica. Revista Brasileira de Fruticultura, v.30, p.1022-1029, 2008.

CHAVARRIA, G.; SANTOS, H.P. dos; SÔNEGO, O.R.; MARODIN, G.A.B.; BERGAMASCHI, H.; CARDOSO, L.S. Incidência de doenças e necessidade de controle em cultivo protegido de videira. Revista Brasileira de Fruticultura, v.29, p.477-482, 2007.

COOMBE, B.G. Growth stages of the grapevine: adoption of a system for identifying grapevine growth stages. Australian Journal Grape and Wine Research, v.1, p.104-110, 1995.

DETONI, A.M.; CLEMENTE, E.; FORNARI, C. Produtividade e qualidade da uva 'Cabernet Sauvignon' produzida sob cobertura de plástico em cultivo orgânico. Revista Brasileira Fruticultura, v.29, p.530-534, 2007.

FELDBERG, N.P.; REGINA, M. de A.; DIAS, M.S.C. Desempenho agronômico das videiras 'Crimson Seedless' e 'Superior Seedless' no Norte de Minas Gerais. Pesquisa Agropecuária Brasileira, v.42, p.777-783, 2007.

FERREIRA, M.A.; PEDRO JÚNIOR, M.J.; SANTOS, A.O.; HERNANDES, J.L. Modificação parcial do ambiente de cultivo da videira 'Cabernet Sauvignon' sobre diferentes porta-enxertos: efeito sobre a produção e o teor de sólidos solúveis. Bragantia, v.63, p.439-445, 2004.

HASELGROVE, L.; BOTTING, D.; HEESWIJCK, R. van; HOJ, P.B.; DRY, P.R.; FORD, C.; LAND, P.G.I. Canopy microclimate and berry composition: the effect of bunch exposure on the phenolic composition of Vitis vinifera L. cv. Shiraz grape berries. Australian Journal of Grape and Wine Research, v.6, p.141-149, 2000.

HENRY, H.A.L.; AARSSEN, L.W. On the relationship between shade tolerance and shade avoidance strategies in woodland plants. Oikos, v.80, p.575-582, 1997.

INTRIGLIOLO, D.S.; CASTEL, J.R. Effects of irrigation on the performance of grapevine cv. Tempranillo in Requena, Spain. American Journal of Enology and Viticulture, v.59, p.30-38, 2008.

KARAGIANNIS, S.; ECONOMOU, A.; LANARIDIS, P. Phenolic and volatile composition of wines made from Vitis vinifera cv. Muscat lefko grapes from the Island of Samos. Journal of Agricultural and Food Chemistry, v.48, p.5369-5375, 2000.

LÓPEZ-MIRANDA, S. Componentes del rendimiento en cv. Verdejo (Vitis vinifera $\mathrm{L}$.), sus relaciones y su aplicación al manejo de la poda. 2002. 274p. Tese (Doutorado) - Universidad Politécnica de Madrid, Madrid.

MANFROI, L.; MIELE, A.; RIZZON, L.A.; BARRADAS, C.I.N.; SOUZA, P.V.D. de. Evolução da maturação da uva Cabernet Franc conduzida no sistema lira aberta. Ciência Agrotécnica, v.28, p.306-313, 2004. 
MOTA, C.S.; AMARANTE, C.V.T. do; SANTOS, H.P. dos; ZANARDI, O.Z. Comportamento vegetativo e produtivo de videiras 'Cabernet Sauvignon' cultivadas sob cobertura plástica. Revista Brasileira de Fruticultura, v.30, p.148-153, 2008.

MULLINS, M.G.; BOUQUET, A.; WILLIAMS, L.E. Biology of the grapevine. Cambridge: Cambridge University Press. 1992. $239 \mathrm{p}$.

NOGUCHI, K.; SONOIKE, K.; TERASHIMA, I. Acclimation of respiratory properties of leaves of Spinacia oleracea L., a sun species, and of Alocasia macrorrhiza (L.) G. Don., a shade species, to changes in growth irradiance. Plant and Cell Physiology, v.37, p.377-384, 1996.

NOVELLO, V.; PALMA, L. de. Growing grapes under cover. Acta Horticulturae, v.785, p.353-362, 2008.

OJEDA, H.; DELOIRE, A.; WANG, Z.; CARBONNEAU, A. Determinación y control del estado hídrico de la vid: efectos morfológicos y fisiológicos de la restricción hídrica en vides. Viticultura/Enologia Professional, v.90, p.27-43, 2004.

PALMA, L. de; NOVELLO, V.; TARRICONE, L. Changes of solar radiation and air $\mathrm{CO}_{2}$ concentration: effects on ecophysiological activity, vine growth and production in table grape grown under protected conditions. In: MEETING STUDY GROUP FOR VINE TRAINING SYSTEMS, 11., 1999, Marsala. Proceedings. Marsala: Università degli Studi di Palermo, 1999. v.2. p.711-717.

PEDRO JÚNIOR, M.J.; HERNANDES, J.L.; TECCHIO, M.A.; PEZZOPANE, J.R.M. Influência do sistema de condução no microclima, na produtividade e na qualidade de cachos da videira 'Niagara Rosada', em Jundiaí-SP. Revista Brasileira de Fruticultura, v.29, p.313-317, 2007a.
PEDRO JÚNIOR, M.J.; PEZZOPANE, J.R.M.; HERNANDES, J.L.; LULU, J.; CASTRO, J.V. de. Avaliações microclimáticas e das características de qualidade da uva de mesa 'Romana' com proteção individual dos cachos. Bragantia, v.66, p.165-171, 2007b.

RANA, G.; KATERJI, N.; INTRONA, M.; HAMMAMI, A. Microclimate and plant water relationship of the "overhead" table grape vineyard managed with three different covering techniques. Scientia Horticulturae, v.102, p.105-120, 2004.

R-PROJECT. R: a language and environment for statistical computing. Disponível em: <http://www.r-project.org/>. Acesso em: 1o dez. 2007.

SCHNEIDER, R.; RAZUNGLES, A.; AUGIER, C.; BAUMES, R. Monoterpenic and norisoprenoidic glycoconjugates of Vitis vinifera L. cv. Melon B. As precursors of odorants in Muscadet wines. Journal of Chromatography A, v.936, p.145-152, 2001.

SEGOVIA, J.F.O.; ANDRIOLO, J.L.; BURIOL, G.A.; SCHNEIDER, F.M. Comparação do crescimento e desenvolvimento da alface (Lactuca sativa L.) no interior e no exterior de uma estufa de polietileno em Santa Maria, RS. Ciência Rural, v.27, p.37-41, 1997.

WEYAND, K.M.; SCHULTZ, H.R. Light interception, gas exchange and carbon balance of different canopy zones of minimally and cane-pruned field-grown Riesling grapevines. Vitis, v.45, p.105-114, 2006.

WHEELER, W.S.; FAGERBERG, W.R. Exposure to low levels of photosynthetically active radiation induces rapid increases in palisade cell chloroplast volume and thylakoid surface area in sunflower (Helianthus annuus L.). Protoplasma, v.212, p.38-45, 2000.

Recebido em 24 de junho de 2008 e aprovado em 30 de janeiro de 2009 